\title{
Impact of chronic low-level ionising radiation exposure on terrestrial invertebrates
}

\author{
J.L. Hingston ${ }^{1}$, M.D. Wood ${ }^{1}$, D. Copplestone ${ }^{2}$ and I. Zinger ${ }^{3}$ \\ ${ }^{1}$ School of Biological Sciences, University of Liverpool, Liverpool L69 3BX, UK \\ ${ }^{2}$ Environment Agency, Richard Fairclough House, Knutsford Road, \\ Warrington WA4 1HG, UK \\ ${ }^{3}$ Swedish Radiation Protection Authority, (SSI), 17116 Stockholm, Sweden
}

\begin{abstract}
There is a need to confirm that the environment is being adequately protected from the contaminants released into it. In the field of environmental radioactivity, modelling and assessment tools are being developed to assess the impact of ionising radiation on wildlife. There is also a need to fill gaps in the scientific data upon which these assessments are based. New documentation has been produced by the UK Environment Agency to provide guidelines on structuring experiments (using environmentally relevant chronic doses) and on selecting suitable non-human species and endpoints for study. It is anticipated that this documentation will be used to direct future experiments. This paper presents the results of the first of these experiments on chronic low-level radiation exposure on soil fauna. Soil fauna species the earthworm Eisenia fetida and the woodlouse Porcellio scaber were segregated and continuously exposed to one of six radiation doses (background, 0.2, 0.4, 1.5, 4.0 and $8.0 \mathrm{mGyh}^{-1}$ ) for a total of 16 and 14 weeks respectively. The endpoints of mortality, number of viable offspring and average weight of an individual were recorded. This paper presents the results for Porcellio scaber.
\end{abstract}

\section{INTRODUCTION}

There is a statutory need to confirm that the environment is protected from the harmful effects of contaminants released into it. The Environment Agency (EA) in the UK has responsibilities, under the Habitats Regulations for England and Wales, for assessing the environmental impact of authorised discharges of contaminants, including radioactive contaminants. The requirement to assess the impact of ionising radiation is now common to all EC member states and to facilitate this the EC-funded Framework for Assessment of Environmental Impact (FASSET) project (FIGE-CT-2000-00102) has collated published data on the effects of radiation on plants and animals and grouped them under four umbrella endpoints (morbidity, mortality, reproductive capacity and mutation) [1]. Following this the FASSET project has now highlighted major gaps in scientific knowledge concerning dose response relationships for particular endpoints for a number of wildlife groups [2]. For example, data is lacking on morbidity for amphibians and reptiles and little data exists on morbidity, mortality, reproductive capacity and mutation for soil fauna [1].

The majority of data collated through FASSET are for wildlife subjected to acute exposures [1]. Most of the data for soil fauna is derived from field experiments undertaken after a nuclear accident or where soil activity concentrations have been artificially increased to replicate acute exposures in the laboratory. It is now recognised that chronic radiation exposure is the most relevant form of radiation exposure in terms of environmental protection and regulation as there are natural as well as anthropogenic sources in the form of authorised waste discharges that give rise to this type of exposure to biota. In response to this lack of information on the effects of low-level chronic exposure 
to biota, the EA commissioned a handbook 'Developing experimental protocols for chronic irradiation studies on wildlife' R \& D Technical Report P3-101/SP2 [3]. The guidelines set down by this document specifically aim to facilitate and direct studies on the effects of ionising radiation on wildlife. As a result it recommends that the dose range for further investigation of effects on soil fauna should encompass $1000 \mu \mathrm{Gyh}^{-1}\left(1 \mathrm{mGyh}^{-1}\right)$ to $5000 \mu \mathrm{Gyh}^{-1}\left(5 \mathrm{mGyh}^{-1}\right)$.

The handbook also recommends which species to study in various wildlife groups and for soil fauna, apart from the extensive use of the earthworm Eisenia fetida in toxicological studies [4-5], the woodlouse is a strong candidate for the following reasons: Woodlice are easily identifiable, widespread, key components in the regulation of decomposition and nutrient recycling in soil ecosystems, easy to maintain in the laboratory and require little space [6]. They have been also used for standard toxicity testing as well as for ecotoxicology and chronic irradiation studies [6-7].

The EA handbook also advocates the use of reproductive endpoints for assessment, as successful environmental protection requires the maintenance of ecosystem function [3]. This function is inherently linked to the success of organisms at a population level therefore any reduction in reproductive success or reproductive capacity could impact on the ecosystem. However the need to observe additional endpoints has also been documented, namely mortality and morbidity (for example, differences in physical appearance and the weight of individuals) [3]. For field studies it has already been concluded that the use of complimentary biomarker techniques combined with methods that relate to organism fitness and site chemistry will provide the most profound data [8]. Applying the same multifaceted assessment to laboratory studies, therefore, would make the results more comparable. Studies on histopathological alterations in the hepatopancreas of woodlice as indicators of environmental stress have already been undertaken. For example, Odendaal and Reinecke [9] studied alterations in histological sections of hepatopancreatic tissue from Porcellio laevis as a biomarker of cadmium exposure. It was revealed that exposure to cadmium sulphate could change the structure of the hepatopancreas. This work and others [10] lend support to the inclusion of histopathological techniques in this study alongside the techniques for measuring reproductive endpoints, mortality and growth.

This paper provides data that starts to address the knowledge gaps highlighted by the FASSET project concerning chronic radiation exposure on soil fauna and begins to derive empirical data for the effects of environmentally relevant radiation doses on morbidity, mortality and reproductive capacity in Porcellio scaber using the approach outlined in the EA handbook [3].

\section{METHODS}

\subsection{Setting up tanks}

Commercially supplied bark compost was dried and the water holding capacity derived. The compost was then homogenised and 500g of dried bark compost rehydrated with $500 \mathrm{ml}$ of distilled water to gain $50 \%$ moisture content ( $0.5 \mathrm{~kg}$ final wet weight). The rehydrated bark compost was placed in a $3.5 \mathrm{l}$ tank. This was repeated for 72 tanks. $10 \mathrm{~g}$ of bran were then layered over the surface of each tank. A length of plywood $(15 \mathrm{~cm} \times 10 \mathrm{~cm})$ was then added to each tank to provide cover for the woodlice.

\subsection{Dosimetry}

The tanks were divided into 6 dose rate groups (12 tanks in each group). The six dose rates chosen for this experiment were background, $0.2,0.4,1.5,4$ and $8 \mathrm{mGyh}^{-1}$. To verify the dose rates the woodlice actually received the dose was measured at three positions along each bench the tanks were located using thermoluminescent dosimeters (four readings per position). The final dose rates received by each group of woodlice were $0.2,0.4,1.5,2.8$ and $8.9 \mathrm{mGyh}^{-1}$. 


\subsection{Test animals}

Woodlice were supplied by Blades Biological Ltd, UK. Fifteen woodlice were introduced into each tank. In accordance with sexual differentiation described by Oliver and Meechan [11], the animals were sexed and weight measurements taken before introduction into the tanks.

\subsection{Duration of irradiation}

The tanks were continuously irradiated with gamma rays for 96 days using ${ }^{137}$ Cs sources (three per bench). The only interruption to radiation exposure was when the tanks were brought out for assessment. The total doses received by each experimental group were 0.5, 0.9, 3.4, 6.4 and 20.4 Gy.

\subsection{Assessment of reproductive, growth and mortality parameters}

At two-week intervals each tank was removed from the room for assessment. The plywood was removed and the bark compost of the tank hand sifted to search for the original woodlice and any progeny that may be present. Each of the woodlice were removed from the tank, weighed and the number of progeny recorded. Any mortality, which occurred, were also recorded. After assessment, the bark compost, plywood and woodlice were returned to their tank and placed back in the irradiation facility.

During the experimental phase (week 8 out of 14 weeks in total) 36 of the 72 tanks were removed for an interim kill. After the weight measurements were taken a proportion of the woodlice were put into $10 \%$ buffered formalin ready for histopathological study. This step was repeated in the final week for a proportion of the remaining woodlice.

\subsection{Histopathology}

The woodlice subject to $10 \%$ buffered formalin were sectioned into three blocks, one transverse section at the $3^{\text {rd }} / 4^{\text {th }}$ segment from the perion, one transverse section anterior to the pleopods on the abdomen and one transverse section to the posterior. The tissues were embedded in paraffin using a Bayer Tissue-Tek III. Sections of tissue, $5 \mu \mathrm{m}$ in thickness, were stained with Haematoxylin \& Eosin stain using a Varistainer. The following tissues; cuticle, gastrointestinal tract, nerve cord, gonads, brood pouch, pleopods and muscloskeletal system, were then examined for signs of hyperplasia, necrosis and neoplasia, using a Leitz Laborlux 11 microscope.

\section{RESULTS}

\subsection{Morbidity}

\subsubsection{Growth measurements}

The general trend was of an increase in average weight over time for woodlice from all dose groups (Figure 1). The only apparent anomaly to this trend was the data for the woodlice that were exposed to a dose rate of $0.2 \mathrm{mGyh}^{-1}$ and background level for week 0 . Upon examination, 
woodlice from the background and $0.2 \mathrm{mGyh}^{-1}$ dose rate groups were not obviously larger than their counterparts in other dose rate groups and as it was at the start of the study none of the individuals had been exposed to any levels of radiation. In subsequent weeks the mean weight values of the woodlice from the background and the lowest dose rate groups were comparable with the rest of the data produced for the other dose rate groups.

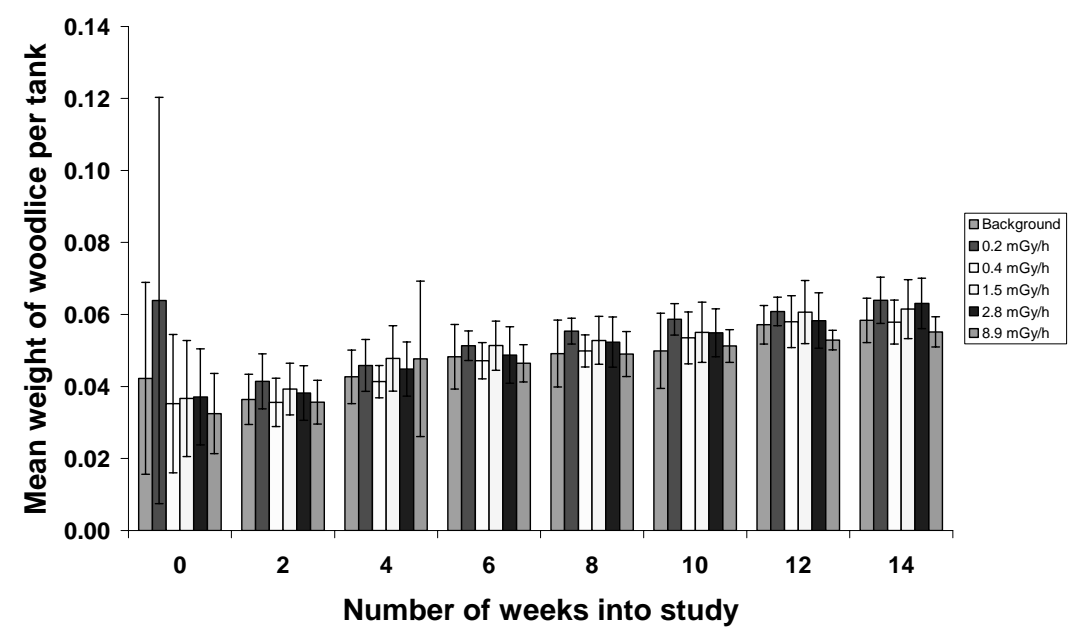

Figure 1. The mean weight of woodlice per tank per dose group over the course of the study ( \pm 1 Standard Deviation).

\subsubsection{Histopathology}

None of the woodlice from any of the dose rate groups analysed showed signs of pathological anomalies. In addition no signs of infection, which can also be a potential indicator of contaminant induced immunosupression [12], were apparent.

\subsection{Mortality}

Figure 2 depicts the cumulative number of woodlice mortalities per dose rate group. No obvious relationship between dose rate and cumulative mortality can be observed.

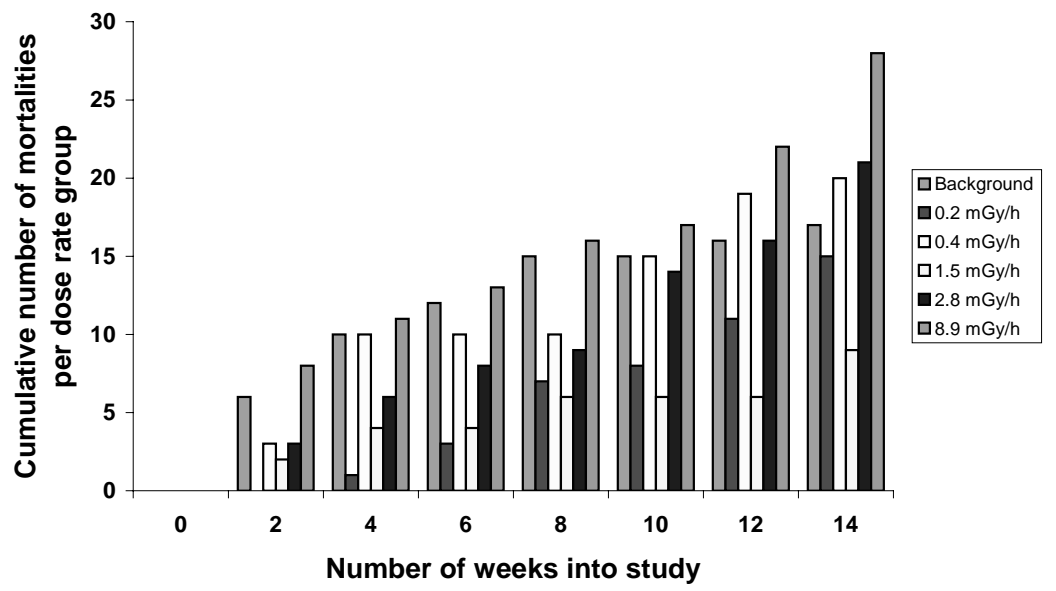

Figure 2. The cumulative number of mortalities per dose group for all tanks for the duration of the experiment. 


\subsection{Reproduction}

\subsubsection{Number of offspring}

The numbers of offspring increase for all dose rate groups up to 4 weeks after commencement of the study (Figure 3). After week 4 the general trend for all dose rate groups is a decrease in the number of offspring (even though no observations of offspring mortality were recorded). In week 14, however, the numbers of offspring for dose rate groups background, 0.2, 0.5, 1.5 and $2.6 \mathrm{mGyh}^{-1}$ increased from those recorded in week 12.

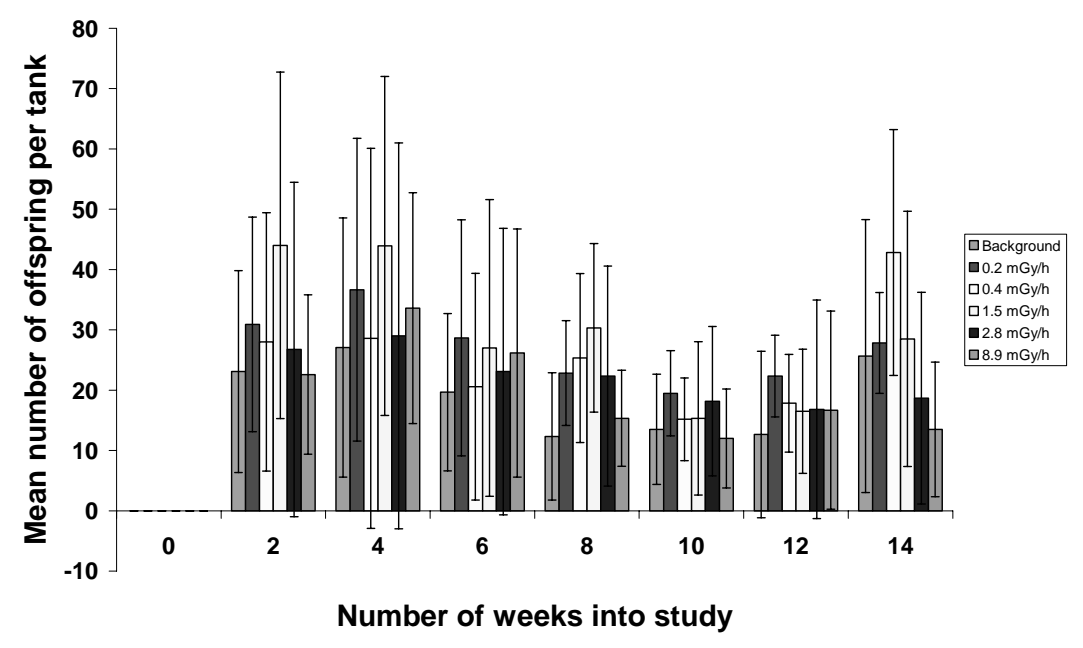

Figure 3. Graph depicting the mean number of woodlice offspring per tank ( \pm 1 Standard Deviation).

\section{DISCUSSION}

It has previously been reported that the general consensus for most studies on biota is that the threshold for statistically significant effects on individual organisms is about $100 \mu \mathrm{Gyh}^{-1}$ with responses increasing progressively with increased dose rate [13]. However this consensus is founded on the most sensitive species, and radiation exposures that are incurred during the most sensitive lifecycle phase of the organism (usually the embryonic phase). At sites contaminated by regulated releases of radionuclides, existing assessments indicate that in the UK the absorbed dose rates are generally much less than $100 \mu \mathrm{Gyh}^{-1}$ and always less than $1000 \mu \mathrm{Gyh}^{-1}$ [14]. In the present study the maximum dose rate that individuals of Porcellio scaber were exposed to was approximately $8 \mathrm{mGyh}^{-1}\left(8000 \mu \mathrm{Gyh}^{-1}\right)$. The study revealed that Porcellio scaber showed no deleterious effects for the endpoints studied, at this dose rate or below. It is well documented that woodlice are able to accumulate extremely high levels of toxic metals such as lead [15], without harm to themselves, and the findings from the present study suggest that this tolerance extends to radionuclides.

The protective effects of small doses of ionising radiation have also been promoted and discussed [16]. Indeed studies on woodlice have proved that aversion to all ranges of radiation sources is not automatic. Kanao et al. [7] observed that at very low levels of ionising radiation ( $\left.4.5 \mu \mathrm{Gyh}^{-1}\right)$ by a beta emitter woodlice migrated towards the source of the radiation as opposed to retreating from the source. This phenomenon was also observed when the woodlice were in the vicinity of a gamma emitter $\left({ }^{57} \mathrm{Co}\right)$ where the exposure rate was $10 \mu \mathrm{Gyh}^{-1}$ [7]. However, when the woodlice were exposed to higher levels of ionising radiation of either source they moved away from the source. Within this study no benefit to the woodlice from being exposed to lower doses of radiation was observed, 
likewise no detrimental effects were observed in woodlice at higher dose rates. Similar growth and mortality rates, histopathology and number of offspring were observed between woodlice exposed to different dose rates.

Whilst the EA handbook provided suitable guidance on a wide range of issues one important issue for future consideration is that the EA handbook states that the organisms under examination should be kept in optimum living conditions by regulating factors such as diet, temperature and light regime. This ensures that any effects observed are attributable to the contaminant under assessment, and it is also consistent with the internationally adopted approach for carrying out ecotoxicological assessments of chemical contaminants. This does poses a dilemma as it has already been noted that organisms which are kept in the laboratory have, in some instances, been more resistant to the effects of radiation due to their 'protected' environment compared to when kept in their indigenous, naturally stressful habitats [17]. The woodlice in this study appeared to be unaffected by the doses given; however, the organisms were removed from predation, overcrowding, competition for food and extreme conditions. The findings from the current study are therefore subject to a qualification that they may not only indicate a high tolerance of radiation by the woodlouse but may also be characteristic of a 'protected' environment.

\section{Acknowledgments}

The authors are grateful to the Environment Agency for the funding for this work.

\section{References}

[1] FASSET., Radiation effects on plants and animals, D. Woodhead and I. Zinger Eds (Framework for the assessment of environmental impact deliverable 4), (2003) pp. 1-196.

[2] C. Williams., J. Radiol. Protect. 23 465-466 (2004).

[3] M. D. Wood, J. F. Knowles, J. Whittaker, D. Copplestone, H. M. Malcolm, S. Bielby., Developing experimental protocols for chronic irradiation studies on wildlife R \& D Technical Report P3-101/SP2, (Environment Agency, Bristol, 2003) pp. 1-96.

[4] E. Bustos-Obregón, R. I. Goicochea, Asian J. of Andrologyl 4 195-199 (2002).

[5] R. G. Kuperman, R. T. Checkai, M. Simini, C. T. Phillips., Ecotox. and Environ. Saf 57 48-53 (2004).

[6] M. Paoletti, M. Hassall., Agri. Ecosystems and Environ. 74 157-165 (1999).

[7] T. Kanao, Y. Miyachi, T. Yamada., J. Environ. Radioact. 63 199-205 (2002).

[8] S. Anderson, N. Belfiore, F. Harrison, Genotoxic effects in aquatic organisms: Methodologies, field related applications, and the role of genetic diversity, (Atomic Energy Control Board and Environment, Canada, 1998) pp. 1-137.

[9] J. Odendaal, A. Reinecke., Ecotox. and Environ. Saf. 56 319-325 (2003).

[10] N. Znidarsic, J. Strus, D. Drobne., Environ. Toxicol. and Pharm. 13 161-174 (2003).

[11] P. Oliver, C. Meechan, Synopses of the British Fauna (New Series) No. 49: Woodlice, (Crothers FSC publications, Cambridge, 1993) pp. 1-135.

[12] E. A. Dyrynda, R. J. Law, P. E. J. Dyrynda, C. Kelly, R. K. Pipe, N. A. Ratcliffe., Environ. Mar. Ecol. Prog. Series. 206 155-170 (2000).

[13] I. Zinger., "Effects of the individual - an overview", Protection of the environment from the effects of ionising radiation., Stockholm, Sweden 6-10 October 2003., (IAEA, Vienna, 2003) pp. 219-223.

[14] D. Woodhead., Environmental Dosimetry, Protecting Biota from Ionising Radiation., Edinburgh, UK 23 January 2004 (The Society of Radiological Protection, London, 2004) pp 1-6.

[15] M. Paoletti, M. Hassall., Agr. Ecosyst. Environ. 74 (1999) 157-165 (1999).

[16] L. Johansson., European J. Nuc Med and Mol Imaging 30 921-933 (2003).

[17] C. Mothersill., J. Radiol. Prot. 21 94-98 (2001). 